\section{Quantum-well thickness dependence of spin polarization of excitons}

\section{Idrish Miah}

Department of Physics, University of Chittagong, Bangladesh; Queensland Micro- and Nanotechnology Centre, Griffith University, Nathan, Brisbane, Australia

\section{Abstract}

The optical orientation of exciton spins in semiconductor quantum wells (SQWs) was investigated by observing the circular polarization of the photoluminescence (PL). The left/right circularly polarized PL in SQWs was measured. It was found that there is a difference between the two different polarization conditions, which is caused by spin-dependent phase-space filling. The PL polarization was estimated from the signals of the left and right circularly polarized PL and was found to depend on the well thickness of SQWs as well as on the sample temperature. The influence of an electric field on the PL polarization was studied.

\section{Introduction}

Spin is a quantum mechanical property associated with intrinsic angular momentum vector of electrons, and many other elementary particles as well as nuclei and atoms, and has an intrinsic magnetic moment associated with it. Therefore, spin is closely related to magnetic phenomena. As an intrinsic magnetic moment is associated with the spin, electron spin provides an extra degree of freedom, socalled the spin degree of freedom, for the electron to interact with a magnetic field. ${ }^{1}$

The idea of utilizing the electron spin in semiconductor devices leads to the growth of the field spintronics, where both charge and spin degrees of freedom play an important role in realizing operations and functionalities.,3 The basic study dedicated to spintronics is to understand the interaction between the particle spin and its solid-state environments in order to make useful devices based on the acquired knowledge. ${ }^{4}$ This may result in the development of novel electronic devices with advantages of e.g., nonvolatility (data retainer), reduced power consumption (energy saver), smaller size (larger integration density) and faster operation (higher speed).
However, one of the basic requirements necessary in developing semiconductor spintronic devices is the efficient generation (injection) of spins (or spin current) in (into) semiconductor heterostructures such that they can be transported reliably (without loss of the spin polarization) over reasonable distances, i.e. distances that are comparable to the device dimensions. ${ }^{5-9}$ In order to understand this, semiconductor-based heterostructures have been studied. For instance, optically detected magnetic resonance in zero field and in a finite magnetic field was used to study the excitons in type-II GaAs/AlAs quantum wells, where the spectra were analysed using the spin Hamiltonian for the quasi-two-dimensional indirect excitons. ${ }^{5}$ Subsequent investigations for photoexcited carrier dynamics and excitonic energy in quantum dots and for optical orientation of electron spins in semiconductor quantum wells (SQWs) are also present. ${ }^{10-12}$

In the present investigation, we focus on the study of the optical orientation of exciton spins in SQWs by circularly polarized photoluminescence (PL) measurements. Spins are generated in SQWs by exciting their heavy-hole valence bands by a left/right circularly polarized light. ${ }^{13}$ The left and right circularly polarized PL in dependences of well thickness (of SQWs), applied electric field and sample temperature are measured. The PL polarization is calculated from the signals of the left and right circularly polarized PL and is found to depend on the strength of the applied electric field, the size of the SQW as well as on the sample temperature.

\section{Materials and Methods}

Samples used in the present investigation were GaAs quantum wells, grown on the Sidoped GaAs substrate using the MBE method. They were $\mathrm{Al}_{0.3} \mathrm{Ga}_{1-0.3} \mathrm{As} \mathrm{SQWs}$ with different well thicknesses $(6,8,10$ and $12 \mathrm{~nm})$. The barrier width was $\sim 20 \mathrm{~nm}$. A negative external bias with different field strengths (up to $-4 \mathrm{~V}$ ) was applied. For the application of the external bias, the top surface of the sample was coated with a semitransparent electrode. We measured the photoluminescence excited by ps right circularly polarized light pulses of a tunable Ti:sapphire laser with a repetition rate of $76 \mathrm{MHz}$ using a streak camera. The PL spectra of the SQW structures displayed intense excitonic lines with a half-width of $2-3 \mathrm{meV}$. Approximately of the same value was the Stokes shift between the exciton peaks in the absorption and PL spectra. The circularly polarized PL was excited directly to the exciton absorption band under normal incidence and was detected with the small long-wavelength
Correspondence: M. Idrish Miah, Department of Physics, University of Chittagong, Chittagong 4331, Bangladesh.

E-mail:m.miah@griffith.edu.au

Key words: quantum well, conduction-band-electron, circularly polarized photoluminescence, phase-space filling.

Received for publication: 7 July 2011.

Accepted for publication: 29 September 2011.

This work is licensed under a Creative Commons Attribution NonCommercial 3.0 License (CC BYNC 3.0).

(C)Copyright M.I. Miah., 2011

Licensee PAGEPress, Italy

Nanotechnology Development 2011; 1:e5 doi:10.4081/nd.2011.e5

shift $(\sim 2 \mathrm{meV})$ to minimize the polarization losses. The circularly polarized PL was detected in the backward direction. Measurements were done at different temperatures by placing the samples in a temperature-regulated cryostat. The PL polarization was calculated by defining it as the ratio of the difference of the signals of the right and left circularly polarized PL to their sum.

\section{Results and Discussion}

The PL in dependences of the external bias, quantum well thickness as well as of sample temperature was measured for the left and right polarization conditions. There was a difference between the different polarization conditions, which is caused by spin-dependent phase-space filling. ${ }^{14,15}$ The resulting PL polarization, as measured with pulses with the same and opposite circular polarization (signals obtained for left and right circularly polarized light), as a function of the time delay was calculated. The dependence of the PL polarization on the external electric bias is shown in Figure 1. The insert shows a typical PL polarization spectrum as a function of the delay. This was for the bias $1 \mathrm{~V}$ and the maximum polarization is $73 \%$. As seen from the insert, the PL polarization decays in a sub-nanosecond. The results are consistent with those obtained in an earlier investigation. ${ }^{16}$

In the presence of the external bias, the electric field reduces the electron-hole exchange coupling by spatially separating the charges, and as a result, the interaction of the hole spin with phonons becomes stronger than the exchange interaction. This leads to breakage of the coupling between the electron and hole spins. As a consequence, the hole spin exhibits fast relaxation, while the electron 
spin holds its orientation, providing the slow component in the polarization decay. Relaxation can result in the PL depolarization due to a flip of the exciton spin as a whole or independent flips of the electron and hole spins. ${ }^{17,18}$ In the first case, the decay time of the PL polarization is directly determined by the exciton spin relaxation rate. If the carrier spins relax independently, the fast flip of the hole spin does not affect the PL polarization. In this case, the decay of the PL polarization is controlled by the relaxation of the long-lived

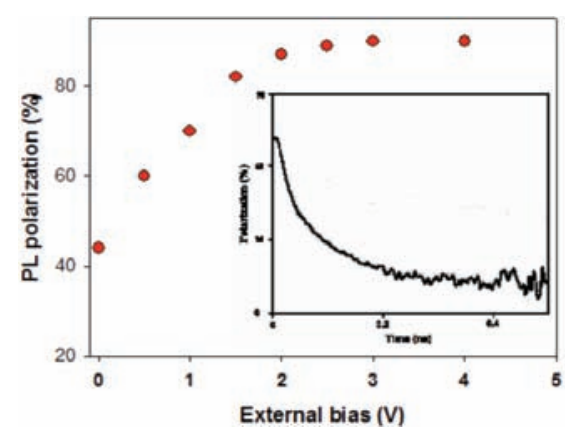

Figure 1. Dependence of photoluminescence polarization on the value of the external bias at $4.2 \mathrm{~K}$. Insert shows a typical PL polarization as a function of the delay. This was for the bias $1 \mathrm{~V}$ and the maximum polarization is $73 \%$.

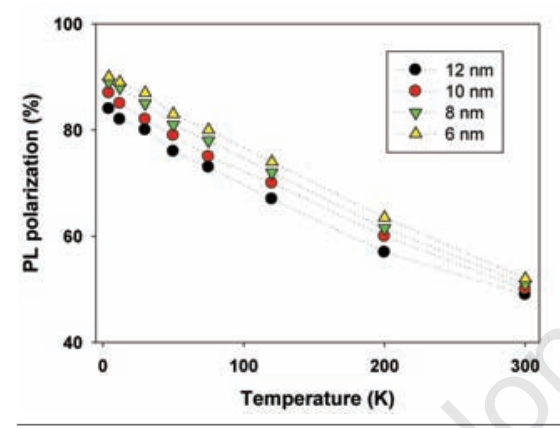

Figure 2. Maximum photoluminescence polarization obtained in the time-dependent measurements as a function of the sample temperature for different quantum well thicknesses.

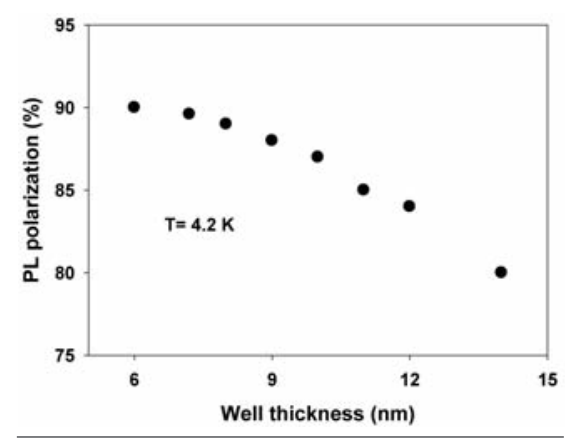

Figure 3. Dependence of the maximum photoluminescence polarization on the quantum well thickness. electron spin. The above analysis reveals that the bias-induced changes in the PL kinetics are related to the transition from dynamics of the exciton spin to that of the independent electron and hole spins. The application of the bias to the SQWs weakens the exchange interaction between the electron and hole spins. This is because the recombination in SQWs is reduced upon the application of a bias due to a decreased wave function overlap of the electrons and holes. In the absence of the bias, the exchange coupling exceeds the spin-phonon interaction originated from photo-induced electron-phonon anharmonicity, ${ }^{19}$ and the main relaxation mechanism is given by the exciton spin flips.

The PL polarization at different temperatures is shown in Figure 2. As can be seen, for all the samples the PL polarization decreases with increasing sample temperature. The observed temperature dependence might be due to the temperature-dependent band-gap shift of the SQW materials. ${ }^{20}$ The temperature dependence of the direct bandgap, determined from the absorption edge, of a zinc-blende semiconductor, such as GaAs, can be described ${ }^{21}$ by $E_{g}(T)=E_{g}(0)-\alpha T^{2} /(\beta+T)$, where $E_{g}(0)$ is the bandgap at $0 \mathrm{~K}$, and $\alpha$ and $\beta$ are temperature independent constant parameters. As the onevalley bandgap gives good agreement for $\mathrm{Al}_{\mathrm{x}} \mathrm{Ga}_{1}$. ${ }_{\mathrm{x}} \mathrm{As}$ with, $x \leq 0.3,{ }^{22}$ the bandgap of the constituents ( $\mathrm{A}$ and $\mathrm{B}$ ) of $\mathrm{A}_{1-\mathrm{B}} \mathrm{B}$ follows the one-valley model and is given by, $E^{A B}{ }_{g}=E_{g}^{A}(1-x) E^{B}{ }_{g}$ $x+\chi(1-x) x$ where $\chi$ is the bowing parameter. ${ }^{23,24}$ As can be seen, the band-gap energy decreases as the sample temperature increases. Thus the circularly polarized light with photon energy slightly lower than the light-hole-band to conduction band energy gap might be able to excite some of the carriers from the light-hole valence band due to the band-narrowing effect at higher temperatures, and consequently the polarization would decrease. The results are consistent with those obtained in another investigation, ${ }^{25}$ where the authors studied the spin relaxation of electrons during transport in the similar material by the PL polarization measurements and showed that the PL polarization under drift increased with decreasing temperature. The observed results also agree with those of the transmission measurements in a bulk sample. ${ }^{26}$

Dependence of PL polarization on the thickness of SQW was studied. The results are shown in Figure 3. As can be seen, the PL polarization decreases as the thickness increases. The breakage of the spin coupling strongly depends on the well thickness, where with increasing thickness of the well, the exchange interaction increases, and consequently, the hole spin relaxation rare decreases. In addition, in thin SQWs, the efficiency of spatial separation of the electron and hole by the electric field is smaller than in thick wells. Owing to this joint action, a relatively small change in the well thickness increases the external bias required for breakage of the coupling of the electron and hole spins. ${ }^{27}$ This fact explains the noticeable changes in the PL polarization and its kinetics, as observed in the experiment (Figure 3). The same effect was observed by others. ${ }^{28}$

\section{Conclusions}

The spin polarization of excitons in the presence of an electric field in SQWs was investigated optically. The left and right circularly polarized PL in dependences of the electric field, sample temperature and well thickness of SQWs were measured. It was found that there is a difference between the two different polarization conditions, caused by spindependent phase-space filling or band filling. The PL polarization, calculated from the signals of the left and right circularly polarized PL, was found to depend on the size of the SQW as well as on the sample temperature. The influence of an applied electric field on the spin orientation was studied. The observed effect was discussed in details.

\section{References}

1. Dyakonov MI, Khaetskii AV. Spin Hall Effect. In: Dyakonov MI, editor. Spin Physics in Semiconductors. Berlin: Springer-Verlag; 2008.

2. Ziese M, Thornton MJ, editors. Spin Electronics. Vol. 569. Heidelberg: SpringerVerlag; 2001.

3. Awschalom DD, Loss L, Samarth N, editors. Semiconductor Spintronics and Quantum Computation. Berlin: Springer; 2002.

4. Miah MI. Semiconductor spintronics and its requirements. JOAM 2008;10:2487-93.

5. van Kesteren HW, Cosman EC, van der Poel WA, Foxon CT. Fine structure of excitons in type-II GaAs/AlAs quantum wells. Phys Rev B Condens Matter 1990;41:528392.

6. Kikkawa JM, Awschalom DD. Resonant spin amplification in n-type GaAs. Phys Rev Lett 1998;80:4313-6.

7. Wolf SA, Awschalom DD, Buhrman RA, et al. Spintronics: a spin-based electronics vision for the future. Science $2001 ; 294$ : 1488-95.

8. Dzhioev RI, Korenev VL, Merkulov LA, et al. Manipulation of the spin memory of electrons in n-GaAs. Phys Rev Lett 2002;88: 256801. 
9. Lou X, Adelmann C, Crooker SA, et al. Electrical detection of spin transport in lateral ferromagnet-semiconductor devices. Nat Phys 2007;3:197-202.

10. Dash SP, Sharma S, Patel RS, et al. Electrical creation of spin polarization in silicon at room temperature. Nature 2009;462:491-4.

11. Ilczuk E, Korona KP, Babiński A, Kuhl J. Spin transport in low-dimensional semiconductor structures. Acta Phys Pol A 2001;100:379-82.

12. Raymond S, Studenikin S, Sachrajda A, et al. Excitonic spin dynamics in semiconductor quantum wells. Phys Rev Lett 2004;92:187402.

13. Pikus GE, Titkov AN. Optical Orientation. In: Meier F and Zakharchenya BP, editors. Modern Problems in Condensed Matter Science. Vol. 8. Amsterdam: Elsevier; 1984.

14. Lampel G. Nuclear dynamic polarization by optical electronic saturation and optical pumping in semiconductors. Phys Rev Lett 1968;20:491-3.
15. Avrutsky IA, Vosmishev AV. Band-filling effect in QWs. Phys Low-Dim Struct 1995;10/11:257-61.

16. Wang T, Li A, Tan Z. Inter-band filling in low-dimensional structures. Proc SPIE 2007;6838:683814-7.

17. Miah MI. Roles of the bias fields in the exchange interaction between the electron and hole spins in GaAs quantum wells. Appl Phys Lett 2009;94:182106-8.

18. Nabi SR. Exchange coupling and interaction. Bull Mater Sci 2000;23:34-7.

19 Kityk IV. Photo-stimulated second harmonic generation in solid films. J NonCrystalline Solids 2001;292:184-92.

20. Varshni Y. Temperature-dependent energy bandgap in semiconductors. Physica 1967;34:149-52.

21. Nag B. Electron Transport. In: Compound Semiconductors. Vol. 11. New York: Springer; 1980.

22. Schubert E. Doping in III-V Semiconductors. Cambridge: Cambridge University Press; 1993.
23. Wang P, Holmes S, Le T, et al. Band-gap in semiconductor heterostructures. Semicond Sci Technol 1992;7:767-70.

24. Casey H, Panish M. Heterostructure Lasers (Part B): Materials and Operating Characteristics. Singapore: Academic Press; 1978.

25. Sanada H, Arata I, Ohno Y, et al. Electron spin transport under drift in GaAs. Appl Phys Lett 2002;81:2788-90.

26. Miah MI. Two-photon spin generation and detection. J Phys D Appl Phys 2009;42: 045503-7.

27. Dacal LC, Brum JA. Binding energy of charged excitons in semiconductor quantum wells in the presence of longitudinal electric fields. Phys Rev B 2002;65:1153249.

28. Heberle AP, Ruhle WW, Ploog K. Quantum beats of electron Larmor precession in GaAs wells. Phys Rev Lett 1994;72:388790. 